\section{Copper Deficiency Myelopathy Can Also Involve the Brain Stem}

Copper deficiency is rare but can result from gastrointestinal surgery, excess zinc in the diet, parenteral nutrition, and malabsorption syndromes. ${ }^{1}$ Copper deficiency gives rise to a sensory ataxic myelopathy, symmetrically involving the pyramidal tracts and dorsal columns in the spinal cord, ${ }^{2}$ producing a clinical picture indistinguishable from subacute combined degeneration (SCD) associated with vitamin $B_{12}$ deficiency. ${ }^{1}$ Brain stem involvement has not been previously reported with copper deficiency. Here we report the first patient with brain stem involvement in copper deficiency myelopathy, demonstrating that it can also be a rhombencephalopathy.

A 47-year-old man with a history of denture cream use insidiously developed bilateral painless vision loss, paresthesias, and quadriparesis over 4 weeks. Neurologic examination revealed left afferent pupillary defect and the presence of optic neuritis. Left upper motor neuron-type facial paralysis, quadriparesis, hyperreflexia, brisk jaw jerk, and a drop sensory level at T6 with graded loss of lower extremity sensation to all modalities were noted. Mild normocytic anemia was present; vitamin $B_{12}$ and serum zinc were within normal levels. Serum copper ( $45 \mathrm{mcg} / \mathrm{dL}$; reference range, $70-140 \mathrm{mcg} / \mathrm{dL}$ ) and ceruloplasmin levels ( $9 \mathrm{mg} / \mathrm{dL}$; reference range, $17-54 \mathrm{mg} / \mathrm{dL}$ ) were low. MR imaging of the cervical spinal cord (Fig 1) and brain (Fig 2) is shown. At 6 months, the patient had not responded to copper supplementation and was wheelchair-bound; bilateral optic atrophy had also set in.

Copper, an essential trace element required by all life forms, is a component of key metalloenzymes (cytochrome c oxidase, superox- ide dismutase, dopamine $\beta$ hydroxylase), which have a critical role in the structure and function of the nervous system. Given its ubiquitous distribution, dietary copper deficiency is rare but results from causes such as gastrointestinal surgery, zinc excess, malabsorption syndromes, and parenteral nutrition. ${ }^{1}$ It is associated with symmetric involvement of the pyramidal tract and posterior columns, resulting in a clinical and radiologic picture indistinguishable from $\mathrm{SCD}^{2}$; rarely peripheral neuropathy and optic neuritis have been described. ${ }^{3}$ Clinical response to treatment is variable. ${ }^{1}$ To our knowledge, this is the first report of a case that shows striking involvement of the pyramidal tracts in the brain stem, in addition to illustrating typical MR imaging changes of copper deficiency myelopathy. It shows that copper deficiency can be a rhombencephalopathy.

\section{References}

1. Kumar N, Gross JB Jr, Ahlskog JE. Copper deficiency myelopathy produces a clinical picture like subacute combined degeneration. Neurology 2004; 63:33-39

2. Kumar N. Copper deficiency myelopathy (human swayback). Mayo Clin Proc 2006;81:1371-84

3. Gregg XT, Reddy V, Prchal JT. Copper deficiency masquerading as myelodysplastic syndrome. Blood 2002;100:1493-95

G. Kumar

M.K. Goyal

S. Lucchese

U. Dhand

Department of Neurology University of Missouri Columbia, Missouri

DOI 10.3174/ajnr.A226 

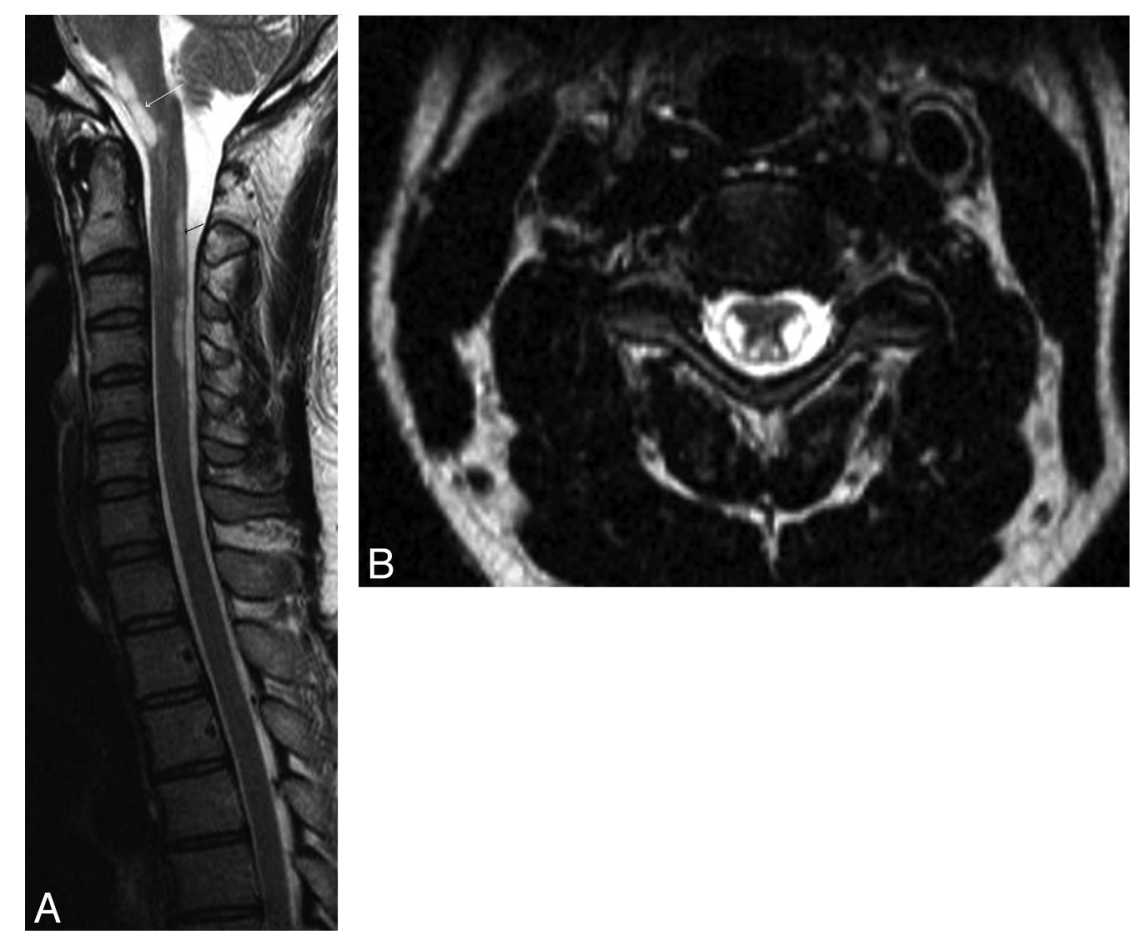

Fig 1. A, Sagittal view through the brain stem and cervical spinal cord shows the extent of T2 hyperintensities involving the pyramidal tract and posterior columns (black arrow), extending along the brain stem (white arrow). B, Axial section through the cervical spinal cord shows the strikingly symmetric T2 hyperintense lateral corticospinal tracts and posterior columns. Thoracic spine MR imaging findings were unremarkable (not shown).
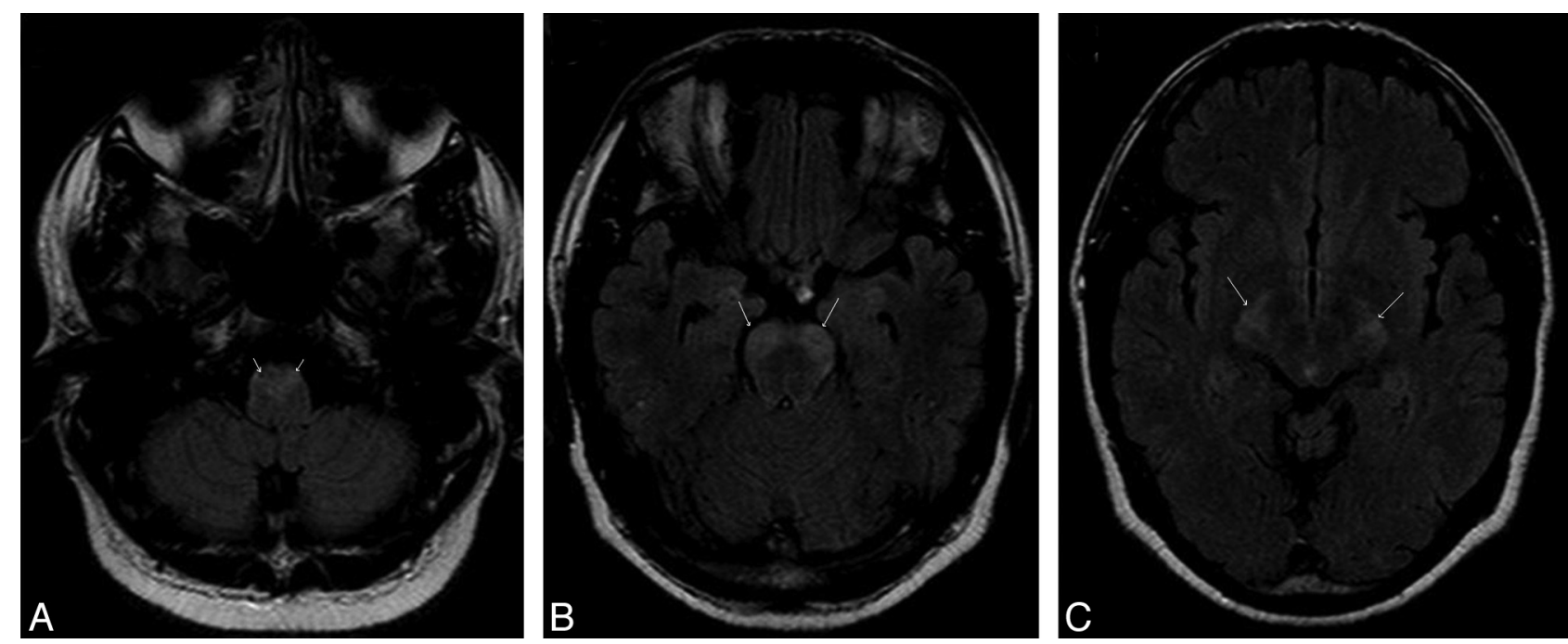

Fig 2. MR images of the brain (axial sections, fluid-attenuated reversion recovery sequences) show the symmetric hyperintensities (arrows) involving the pyramidal tract in the (A) medulla, (B) pons, and (C) midbrain. 